\title{
About the existence of the universe among speculative physics, metaphysics and theism: an interesting overview
}

\author{
Paolo Di Sia* \\ Free University of Bolzano-Bozen, Viale Ratisbona 16 \\ 39042 Bressanone-Brixen, Italy \\ *E-mail address: paolo.disia@gmail.com
}

\begin{abstract}
In this paper an interesting overview about the existence of the universe is given. It will focused in particular on efforts of modern-speculative physics, considering then metaphysical considerations and some ideas of theism.
\end{abstract}

Keywords: Universe; Modern Physics; Speculative Physics; Metaphysics; Cosmology; Theism; Reality

\section{INTRODUCTION}

The argument related to "Why there is something rather than nothing?" is a question that, together with the concept of infinity, sense of life and reality, "destiny" of future, essence of space and time and others, involved in the human history thinkers, scientists, philosophers, theologians, and it is often counted as the "mother of all questions" [1-3].

Considering our knowledges at today, the entire universe, accessible to observations, is the result of a concatenation of causes, well scientifically explainable, which began about fourteen billion years ago with a first event called "big-bang". But what caused the big-bang?

a) Someone says that it makes no sense to ask this question, assuming that time is born with big-bang and before that no previous events have been, shifting so the question about the cause.

b) Others prefer to think the big-bang as an event occurred within a bigger universe, perhaps infinite and eternal. In this broader context, there would be the opportunity to explain the big-bang through a previous cause, but not the fact that there is a bigger universe, nor the infinite series of causes, that led to a particular big-bang, from which began our universe.

c) Because the laws of physics are what they are? Many people consider just these conceptual difficulties for finding the last fortress of theism, with the belief that everything has been created by God; but this moves the problem on God.

In the following I consider salient ideas, research and possible conclusions of modernspeculative physics, metaphysics and faith. 


\section{UNIVERSE AND MODERN-SPECULATIVE PHYSICS}

Modern-speculative physics has been very interested about the question of the existence of universe, both on technical level, and at level of thought. In last years, key concepts and ideas have been considered and developed, around which the intellectual cognitive efforts evolve. Among the main ones, we have:

\subsection{The anthropic principle}

The "anthropic principle" is a cosmological principle based on consideration that all scientific observations are subject to the bonds of our existence as observers. So the observations of physical universe "might be compatible" with the conscious and sapient life that observes them. Some proponents of this principle underline that the anthropic principle explains why universe has the right age and the required fundamental physical constants for accommodate conscious life. It has been later developed as hypothesis that tries to explain the current features of the universe.

The original enunciation of the anthropic principle derived by Brandon Carter, and subsequently reinterpreted from some followers of the so-called "intelligent design", otherwise known as "scientific creationism". Moreover it must not be confused with "parascientific argumentations", developed during the $9^{\text {th }}$ century and beginning of the $10^{\text {th }}$ century. In these periods cosmology was read from a mainly fideist viewpoint, therefore unrelated to the current argumentations of modern science.

The principle has been published by Carter in 1974 in two versions:

a) the so-called "Weak Anthropic Principle": "Our position in space and time is necessarily privileged, as it is compatible with our existence of observers";

b) the so-called "Strong Anthropic Principle": "The universe, and consequently the basic parameters that characterize it, must be such as to allow the creation of observers within it, at a given stage of its existence".

These statements have been reinterpreted, in particular by John D. Barrow and Frank J. Tipler (1986). They enunciated three new versions of the anthropic principle, with divergences from Carter statements:

a) "Weak Anthropic Principle": "The observed values of all physical and cosmological quantities do not have the same probability, but assume values limited by the prerequisite that there are places where carbon-based life can evolve and by the prerequisite that the universe is old enough to have already allowed this".

b) "Strong Anthropic Principle": "The Universe must have those properties, which allow life to develop within it at some point in its history".

c) "Last Anthropic Principle": "It must necessarily develop an intelligent processing of information in the universe, and once appeared, this will never die".

Authors derived the $3^{\text {rd }}$ principle from $2^{\text {nd }}$ one, because it is without sense a universe that has the capacity to produce intelligent life and it is not sufficiently long for developing it.

The statements of Carter, Barrow and Tipler anticipated many contributions and disputes by a lot of scientists, including Stephen Hawking, Paul Davies, John Archibald Wheeler, Leonard Susskind, Steven Weinberg [4,5]. 


\subsection{Universe from nothing}

This idea is based on fact that the universe formed itself spontaneously, as result of quantum fluctuations in which it came into existence from nothing. That is plausible, considering current knowledges about quantum mechanics. The idea is bound to a special solutions set of a mathematical entity, known as the "Wheeler-DeWitt" equation, related to the efforts of theoretical physics for combining the two pillars of modern physics, i.e. quantum mechanics and general relativity, for a unified description of the universe.

In the 1960s the physicists John Wheeler and Bryce DeWitt combined the two previous incompatible ideas in a mathematical framework, currently known as the "Wheeler-DeWitt equation"; it is a particular type of functional differential equation, which emerges from the quantization of the theory of general relativity, expressed according to the canonical formalism.

The core of their thinking rely on Heisenberg's uncertainty principle. This allows a very small empty space to come probabilistically into existence, due to quantum fluctuations; from the viewpoint of theoretical physics, it is the creation of a "metastable false vacuum". When a small bubble of space is created, we have two possibilities:

a) it does not expand rapidly, so it disappears almost instantly;

b) if there is a sufficiently large expansion, then a universe can be created; the small bubble has an exponential expansion, reaching a size in which a universe can form a big bang [6].

\subsection{Universe as a computer simulation}

This proposal considers that our universe could be a numerical computer simulation. It is suggested that our far-evolved descendants may have built such a program for simulating the past and re-creating the conditions of their remote ancestors. Our descendants, from future, would have the computer capacity to run complex simulations, millions of simulations, and millions of virtual universes with billions of simulated brains.

One of the key-points of this argumentation is the fact that, if humanity continues surviving and computer technology continues advancing, we will unavoidably reach the possibility to simulate the entire planet and all humans.

There is a mathematical physics well-established theory, based on the "holographic principle", that states we are living in a hologram, with a size of trillions times smaller than an atom $[7,8]$.

\subsection{Conspiratorial cosmology}

This idea is based on fact that all parameters describing the universe can be produced by a small set of known numbers. This potential conspiratorial origin of the universe shows how the relevant fundamental parameters can be constructed though complex mathematical operations from a small set of "conspiratorial numbers".

Cosmological models give little constraints, but it is not excluded that these small set of numbers can change "ad-hoc" some physical parameters of the universe, with possible dramatic consequences.

A common element of cosmology and conspiracy theory is the "affection to numbers". Of the rest, the entire knowledge of modern cosmology about the universe is related to a set of numbers, the "cosmological parameters", with experimental cosmology, that tries to confirm 
them. The conspiracy theory assumes that some members of the "lodge" are favourable to numbers and communicate them to humans through initiates, as mathematicians, science fiction authors, men considered lunatics [9].

\subsection{The biocentrism}

The laws of physics and chemistry are able to explain the biology of living systems, but a complete understanding of life cannot be found only microscopically looking at cells and molecules.

The "biocentrism" views the world with glasses of subjective experience, with "consciousness". Most of the comprehensive theories of universe don't take into account the crucial factor, that the biological creatures are creating them; in particular biology, among these theories, has a bigger role, as first and last study of science.

The models at the basis of consciousness define the human perception of all reality. In 2007, the pioneer of stem cell research Robert Lanza has developed a theory which postulates that time, space and our entire reality are different to what we always believed to be.

This theory, known as "bio-centrism", describes reality as a process deeply involving our consciousness; without that, all matter is in an indeterminate probability state and time has no "real" existence.

Quantum mechanics is considered, "quantized states" of consciousness; the reality is an endless sea of statistical informations, in which all probabilities simultaneously exist. The laws for realizing our reality are the most probable among the countless ones.

This model provides also an amazing evidence, currently difficult to refuse: it is intrinsically infinite and therefore, statistically, a "life after death" seems to be inevitable. The notion of "external reality" falls; the activity of consciousness, born by our biology, creates the world.

The global experience is an organized whirl of information in our brain. The world appears to be designed for life not just at microscopic scale, but at the level of the universe [10].

\section{UNIVERSE AND METAPHYSICS}

The question about "Why does the universe exist?" has been deeply considered in philosophy, concluding that it is both a soluble and an insoluble enigma.

Among the solutions, Heidegger considered the question as the "fundamental problem of metaphysics" [11].

Gilson sustained that the cause of the universe is a "pure act of existence", absolute and self-sufficient [12].

For Edwards, if it can be a persuasive argumentation the fact that there exists a metaphysical entity transcending and including the universe, then it is possible an answer to the question "Why does the universe exist?", and it is therefore meaningful [13].

Among the second ones, Huxley suggested that we must learn to accept that the universe is an "irreducible mystery".

Russell asserted that there is no ground motivation for the assumption that the universe must have a cause.

Wittgenstein, with the modern logic, Koestenbaum, Waisman and others considered meaningless this question; the query on "why there is something and not nothing" is either ill- 
formed or without benefits, since any comprehensible answer will cause the same question [14].

Some problems about the definition of the universe raised too; the Kaufmann's definition has been considered the orthodox view, i.e. the universe as "all space, along with all the matter and radiation in space", in opposition to others, as the Edwards' definition of the universe as "the totality of things" [15].

A possible answer could come from the hypothesis of many worlds; in this scheme, the problem of "why things are not much better than they are" is different. Things may be better elsewhere, with different injustices with respect to our universe. But in a more negative vision, it could be no connection between goodness and reality; things may exist not because they must exist, but for other reasons, or the universe may be inexplicable. This would make a difference to morality too [16].

\section{UNIVERSE AND THEISM}

Physicists, through calculations and theoretical cosmological modelling, believe that, if some parameters of the universe change also very slightly, the appearance of life would be impossible. Theists add that this is not merely a coincidence; on their viewpoint, God created the universe with these special features, for permitting the possibility of life. The idea of a "God Creator", who with a will act created the universe, is generally rooted in religious cultures.

One of the great difficulties remains the nature of time. St. Augustine, in the $5^{\text {th }}$ century, was aware of the problem of time (causality principle: time must exist before that a thing causes another one). Boethius, a century later, elaborated a more abstract and sophisticated concept of creation, considering the existence of God as "out of space and time", not "before", i.e. a timeless God. He creates the universe in a more complex sense, keeping it in existence at all times, acting "here" and "now".

Further efforts brought during years to creation of three alternatives:

a) God "in time", "cause" of creation.

In this case, there is a sequence of events, with a causal dependence of every step from the previous one, through the physics laws. God would be the first term of this series (Figure $1)$.

\begin{tabular}{|c|c|c|c|c|}
\hline \multicolumn{3}{|c|}{ Physicslaws } & \multicolumn{2}{|c|}{ Physicslaws } \\
\hline God & $\rightarrow$ & ........... & Events (past) & Events (present) \\
\hline
\end{tabular}

Figure 1. Scheme for the possibility: God "in time".

b) God "without time", which continuously "gives existence" to the universe.

If God is "out of time", is not part of this succession of causes, but directly acts; $\mathrm{He}$ is "here" and "now", is the "explanation" of the universe (Figure 2). The universe can begin temporally, or be infinite (the series does not have in this case a first term).

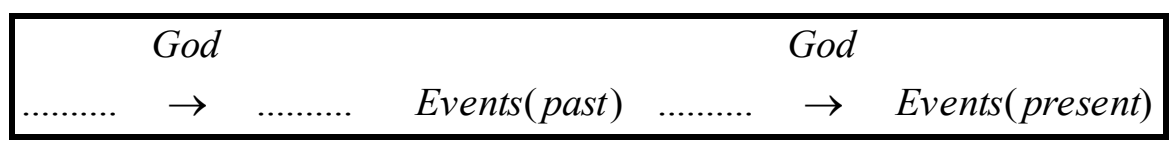

Figure 2. Scheme for the possibility: God "without time". 
c) A third hypothesis could be an "iterative map"; the previous series becomes circular, not necessarily beginning with God (Figure 3). This scheme refers also to the so-called "bootstrap universe"; it contains in itself a justification based on natural and physical interactions [17].

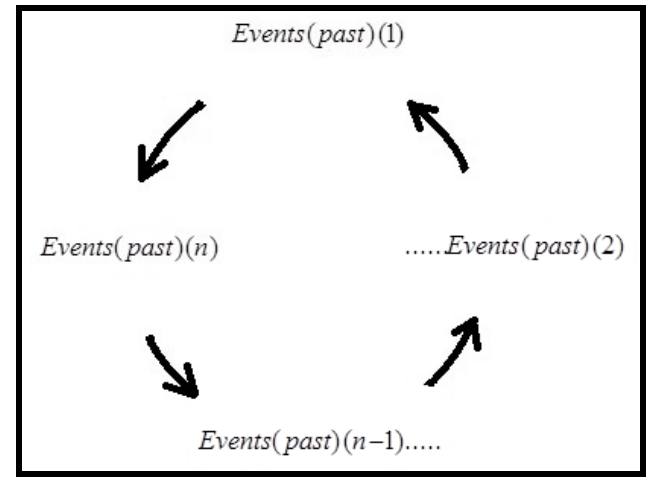

Figure 3. Scheme for a possible "iterative map".

The theist replies that it is more reasonable to attribute the character of necessity to God, as simple conceivable entity, not to the universe, which is complex and particular. To objections that an infinite mind (God) is infinitely complex, and for that less probable than a complex universe, he replies that maybe God is not a mind, but something different, or at least different from our idea of mind. It is possible to assume that the complexity of the universe has been established naturally as a result of normal physical laws, but this moves the problem to the creator of the physical laws.

In an expanding universe, it seems possible that energy can form spontaneously, without attributing the cosmic order to the intervention of God, nor to the introduction of organization at the instant of initial singularity, downloading the responsibility to gravitation, but it moves the problem on the explanation of the manifested order in the gravitational field.

The order depends on whether it is possible to neglect the gravity. Gravity tends to cause the spontaneous creation of structures. In systems, which are not subjected to gravitation, order implies complexity, disorder implies simplicity. For gravitational systems the reverse occurs.

But this still does not solve all problems; if the "state" of the universe is not a problem, it remains to investigate its "laws". Why these laws and not others? Why particles have the characteristics they have, and not others? The theist replies: "God wanted so".

The wide variety of physical laws is a contingent phenomenon, linked to low temperatures. Increasing temperature of universe, the various forces lose their identity, and for temperatures of order of $T=10^{32} \mathrm{~K}$ (one hundred thousand billions of billions of billions of Kelvin degrees), all forces should combine into one only "superforce". The subatomic structures become less complex, and simplier elements appear; also the physics laws and the building blocks of matter would start as a simple structure. But the theist can reply: "Why one superforce only and a few simple structures, and not nothing?".

A singularity is one of the limits of what is knowable by science. From a so-called "naked singularity" can get out anything, without a physical cause, which anticipates the effect. Some cosmologists think that the universe emerged from a similar singularity, without a cause. If true, singularities would constitute the interface between "natural" and "supernatural", being the ultimate unknowable $[18,19]$. 


\section{CONCLUSION}

From a purely empirical point of view, there is the well-founded doubt that the question on "Why is there something rather than nothing?" is simply misplaced, a kind of semantic trap. What evidence do we have about the existence of the "nothing"? And even considering possible the absence of anything, because the "nothing" might be more probable, easier, or more natural than what exists?

In any case, the topics discussed in this overview have always interested thinkers, scientists, philosophers, theologians, believers and simple people who think. On the other hand, perhaps the great existential questions have more to do with the limits of our mental categories, and of our own existence, than with the reality, which is "out" of us.

The considered models-ideas are at the frontier of modern theoretical physics and cosmology. These issues have given and give always new impetus to the debate about God and the existence of the universe.

\section{Biography}

Paolo Di Sia is currently professor of "Foundations of Mathematics and Didactics" by the Free University of Bolzano-Bozen (Italy). He obtained a Bachelor in Metaphysics, a Laurea in Theoretical Physics and a PhD in Mathematical Modelling applied to Nano-Bio-Technologies.

He interested in Classical-Quantum-Relativistic Nanophysics, Theoretical Physics, Planck Scale Physics, Mind-Brain Philosophy, Philosophy of Science, of Physics and of Cosmology, Econophysics.

He is author of more than 140 works at today (articles on national and international journals, scientific international book chapters, books, internal academic notes, scientific web-pages, in press), reviewer of two mathematics academic books and is preparing a chapter for a scientific international encyclopedia. He is reviewer of some international journals and invited to review and as editor. He obtained 4 international awards, is included in Who's Who in the World 2015 ( $32^{\text {nd }}$ Edition), is member of 5 scientific societies and of 13 International Advisory/Editorial Boards.

paolo.disia@gmail.com; http://unibz.academia.edu/PaoloDiSia;

http://it.linkedin.com/pub/paolo-di-sia/23/928/8b7; http://paolodisia.weebly.com/.

\section{References}

[1] P. Di Sia, Describing the concept of "infinity" among art, literature, philosophy and science: a pedagogical-didactic overview, Journal of Education, Culture and Society, N. 1, pp. 9-19 (ISSN 2081- 1640), http://jecs.pl/doi/10-15503-jecs20141-9-19/ (2014).

[2] P. Di Sia, Looking at the Dimension of Time among Science, Psychology and Everyday Reality, International Letters of Social and Humanistic Sciences (ILSHS), N. 1(2), pp. 146-153 (ISSN 2300-2697), http://www.ilshs.pl/wp-content/uploads/2014/09/ILSHS12-2015-146-153.pdf (2015).

[3] P. Di Sia, Approaching youngs to unified theories: the charm of string theories, Procedia - Social and Behavioral Sciences Journal (Elsevier) (2015), in press.

[4] B. Carter, Large number coincidences and the anthropic principle in cosmology, in: "Confrontation of cosmological theories with observational data", Proceedings of the Symposium, Krakow, Poland, September 10-12, 1973, Dordrecht, D. Reidel Publishing Co., pp. 291-298 (1974). 
[5] J. D. Barrow, F. J. Tipler, The Anthropic Cosmological Principle, Oxford University Press, Oxford, $1^{\text {st }}$ Ed., 738 pp., ISBN-13: 978-0192821478 (1988).

[6] L. M. Krauss, A Universe from Nothing: Why There Is Something Rather than Nothing, Free Press, New York, 240 pp., ISBN-13: 978-1451624465 (2013).

[7] N. Bostrom, Are You Living In a Computer Simulation?, Philosophical Quarterly, Vol. 53, N. 211, pp. 243-255 (2003).

[8] P. Di Sia, Extreme Physics and Informational/Computational Limits, Journal of Physics: Conference Series 306, p. 012067, 8 pp., doi:10.1088/1742-6596/306/1/012067 (2011).

[9] J. P. Rachen, U. G. Gahlings, Conspiratorial cosmology - the case against the Universe, Journal of Comparative Irrelevance (Letters), Vol. 23, p. 966 (4 pp.) (2013).

[10] R. Lanza, B. Berman, Biocentrism: How Life and Consciousness Are the Keys to Understanding the True Nature of the Universe, Benbella Books, Dallas, 213 pp., ISBN-13: 978-1935251743 (2010).

[11] M. Heidegger, An introduction to metaphysics, Yale University Press, New York (1959).

[12] E. Gilson, God and philosophy, Yale University Press, New Haven (1941).

[13] P. Edwards, Why?, in: P. Edwards \& A. Pap (Eds.), "A modern introduction to philosophy”, The Free Press, New York, $3^{\text {rd }}$ Ed., pp. 796-810 (1973).

[14] L. Wittgenstein, Tractatus Logico-Philosophicus, Dover Publications, New York, 471 ${ }^{\text {st }}$ Ed., 128 pp., ISBN-13: 978-0486404455 (1998).

[15] W. J. Kaufmann, Universe, W. H. Freeman and Company, New York, $3^{\text {rd }}$ Ed. (1991).

[16] K. J. Kraay, The Theistic Multiverse: Problems and Prospects, in: Y. Nagasawa (Ed.), "Scientific Approaches to the Philosophy of Religion", Palgrave MacMillan, Houndsmills, pp. 143-162 (2012).

[17] R. T. Cahill, C. M. Klinger, Pregeometric modelling of the spacetime phenomenology, Physics Letters A, Vol. 223, Issue 5, pp. 313-319 (1996).

[18] P. Di Sia, Exciting Peculiarities of the Extreme Physics, Journal of Physics: Conference Series, 442(1), p. 012068 (6 pp.), doi:10.1088/1742-6596/442/1/012068 (2013).

[19] P. Davies, God and the New Physics, Simon \& Schuster, New York, ISBN-13: 9780671528065 (1984). 